Thorax (1968), 23, 136.

\title{
Bronchial carcinoma
}

\author{
B. T. LE ROU X ${ }^{1}$ \\ From the Thoracic Unit, Edinburgh
}

From a retrospective review of 4,000 patients with bronchial carcinoma, managed in the Thoracic Surgical Unit in Edinburgh, the salient features of presentation, surgical management, and the natural history in those unsuitable for surgical management are reviewed.

From a retrospective analysis of the case records of 4,000 patients managed over a period of 15 years and established sooner or later to have bronchial carcinoma, it is possible to make certain generalizations.

Bronchial carcinoma is more common in men ; only $10 \%$ of patients are women. The highest incidence of the disease was in the sixth and seventh decades; $10 \%$ of patients were 70 years or older when they were first seen ; about one-fifth of women and fewer than one-sixth of men with bronchial carcinoma were younger than 50 years at the time of their first investigation. While the yearly incidence of the disease did not appear to be changing significantly, the incidence among those over 70 years was rising and among those under 50 years might have been falling. An increasing incidence of the disease in the aged was probably explicable on the grounds of a generally aging population; a diminishing incidence in the younger age group is recorded in many series, and an explanation is lacking.

The survey, of which this is a summary, was made in a region where coal mining is a common occupation, and of the patients in this series $15 \%$ were coal-miners, an occupational incidence which is disproportionately high. It is generally maintained that coal mining does not predispose specifically to bronchial carcinoma. That a large number of coal-miners were found to have bronchial carcinoma may be related to the fact that theirs is an occupational group regularly examined radiographically and probably more often than are most other occupational groups.

Of every four patients with bronchial carcinoma, three were known to have been cigarette smokers, and most of these were heavy smokers. Of the total number of patients, $3 \%$ smoked only a pipe and $4 \%$ claimed never to have

1Present address: Thoracic Unit, Wentworth Hospital, Durban, Natal, South Africa smoked: among the women in the series $15 \%$ claimed never to have smoked. Latterly there emerged a noticeable reluctance among patients to admit that they smoked, and it is now commonplace for patients, when asked the question 'Do you smoke?' to answer, quite honestly, that they do not on the grounds that they had stopped smoking with the onset of symptoms, or with the finding of an abnormality on a chest radiograph, or even on the way to hospital on the morning of interrogation. In one-third of those who did not smoke the tumour was squamous. The smoking habits of $20 \%$ of the patients were not known, an incidence of omission in the records probably significant of the percentage of failure adequately to record in all other aspects of the anamnesis, and perhaps an argument in favour of demanding, from rotational junior staff who interrogate patients, the completion of a detailed pro forma rather than the recording of answers to those variable questions which they may remember to ask. While it has been established that a large proportion of those who developed bronchial carcinoma had smoked cigarettes for some years, the precise relationship between cigarette smoking and bronchial carcinoma was not established. It is clear that not smoking is not a guarantee against the development even of squamous carcinoma, and that cigarette smoking is not the only factor in the genesis of bronchial carcinoma.

Patients shown by investigation to have bronchial carcinoma presented in one of five ways: (1) with one, some, or all of the cardinal symptoms of respiratory disease-cough, sputum, haemoptysis, chest pain, dyspnoea, and wheeze : (2) without symptoms and because of an abnormality detected on a chest radiograph made for routine purposes during mass miniature radiographic surveys; during radiographic surveys among coal-miners and other occupational groups; during the routine follow-up of patients 
known to have had pulmonary tuberculosis ; or for insurance or immigration purposes; (3) because of evidence of extension of or metastases from bronchial carcinoma, e.g., cerebral or osseous metastases, hoarseness of voice, obstruction of the superior vena cava, or jaundice; (4) with non-specific symptoms such as loss of weight and appetite, insomnia, and dyspepsia ; (5) with what may be called the humoral or neural concomitants of the disease-hypertrophic pulmonary osteoarthropathy, myopathy, and neuropathy; and others such as Cushing's syndrome.

Cough was a symptom in three of every four patients with bronchial carcinoma, and in $40 \%$ cough was severe and one of the primary reasons for medical advice being sought. Cough was, in nearly all instances, at some time productive. In many patients cough was not a new symptom and was often attributed to smoking. Worsening of an otherwise chronic cough, often in relation to one or several febrile episodes, was common.

Haemoptysis was a symptom in more than half $(57 \%)$, and in $4 \%$ of patients haemoptysis was the only symptom. Presentation for investigation in $1 \%$ was prompted by a single haemoptysis. In patients with haemoptysis this symptom varied from occasional to daily. The complaint of daily haemoptysis-usually in the form of bloodstreaking of the sputum in the early morningwas regarded as very suggestive of bronchial carcinoma. There was commonly delay, even among doctors later shown to have bronchial carcinoma, of months rather than weeks before patients with the complaint of haemoptysis presented for investigation. Delay in investigation of haemoptysis by a thoracic surgeon was often occasioned both by delay in referral for a surgical opinion and delay on the part of the patient to report the abnormality.

Pain related to bronchial carcinoma was not usually acute pleural pain and was commonly an intermittent ache, which lasted for hours at a time, was worse at night, and was aggravated by posture and relieved by activity. Severe pain was common in those patients shown by investigation to have invasion of the chest wall, but chest wall invasion, vertebral invasion, and rib and vertebral metastases were found in patients who denied pain, and the complaint of pain was not uncommon in patients found to have pulmonary tumours which were successfully managed surgically and in whom pain could not be related to pleural invasion or infection.

It was often not possible to equate the complaint of dyspnoea with diminution in measured respiratory function; very nearly normal respiratory function figures were recorded in patients among whose more distressing complaints was dyspnoea; there was little relationship between the size of a radiographic opacity (and presumably, therefore, the extent of deprivation of functioning lung) and the severity of the dyspnoea.

One in every four patients with bronchial carcinoma gave a history either of an acute febrile respiratory illness, often called influenza, from which recovery had been slow and incomplete, or of a series of acute febrile illnesses with complete or incomplete recovery between episodes. Cough with purulent sputum, chest pain, dyspnoea, and haemoptysis were often features of these acute illnesses, but evanescent features of which little cognizance had been taken during the acute episode; in fact these features were taken for granted as part of the acute episode. This history-of failure to make an anticipated recovery from an acute respiratory illness-is accepted as strongly suggestive of the diagnosis of bronchial carcinoma, as is daily haemoptysis.

Wheeze was a presenting symptom in $2 \%$ of patients, and in those who were not chronic asthmatics the complaint of wheeze could usually be related to a tumour that had extended in a main bronchus close to the main carina, and even into the trachea; and, as a prognostic feature, wheeze, like stridor, was often significant of inoperability. Stridor, as distinct from wheeze, was less common, and fewer than $1 \%$ of patients were admitted to hospital urgently stridulous and in need of therapeutic bronchoscopy, at which the tumour had to be cored from the trachea or both main bronchi in order to establish a more adequate airway. While stridor with bronchial carcinoma is often a herald of death, acute tracheo-bronchitis, even in an adult, may present with stridor as evidence of gross proximal airway obstruction and is always an indication to investigate stridor by bronchoscopy.

There was a group of patients- $15 \%$ of the total - who presented not with respiratory symptoms but with symptoms such as loss of weight and appetite, tiredness and lassitude, non-specific general ill-health, dyspepsia, with recent difficulty in the control of previously easily managed diabetes, and so on. In these, referral to a thoracic surgical unit was usually the result of an abnormality having been detected on a chest radiograph made as one of many routine investigations undertaken in a search for a cause for non-specific symptoms, or because an abnormality 
had been recognized during routine fluoroscopy of the chest as part of a barium study.

Nearly $2 \%$ of patients with bronchial carcinoma had at the time of their first presentation the complaint of dysphagia, and in half of these dysphagia was the presenting complaint. Dysphagia from malignant disease is nearly always the consequence of a primary alimentary tumour : but where it is the consequence of metastatic malignant disease the primary tumour is nearly always bronchial.

A small group of patients presented with the complaint of recurrent peripheral venous thromboses, often called thrombophlebitis migrans. In this group the incidence of operability was lower than average; the incidence of metastases at operation was higher than average; the incidence of death from pulmonary embolism, in convalescence from an operation, high ; and that of long survival, low.

Bronchial carcinoma was found in patients in whom presentation was a myopathy or neuropathy ; because of Cushing's syndrome ; because of exfoliative dermatitis, dermatomyositis, or purpura; and with other evidences of general systemic disease. A patient who presents with spontaneous pneumothorax or an inguinal hernia and a chronic cough may well have a bronchial carcinoma. Cough syncope and cough fractures may be the form of presentation in men.

Apart from a small number of patients, too ill at the time of admission to hospital for routine investigations or who died before investigations could be completed, all patients were submitted to a routine series of investigations. The history and clinical examinations were recorded. Routine chest radiographs were made and all patients well enough to go to the radiology department were examined fluoroscopically, primarily for evidence of phrenic paresis and displacement of the barium-filled oesophagus. Tomograms and other special films were made, in circumstances in which they were believed necessary, for diagnostic or record purposes. Bronchoscopy was a routine and it was the practice to make a biopsy from a visible tumour. In $58 \%$ of patients the diagnosis of bronchial carcinoma was confirmed at bronchoscopy. The tumours in these patients were called 'central' on the ground of visibility at bronchoscopy. In the remaining patients the appearances at bronchoscopy were normal, or bronchi were displaced but with the mucosa normal, and these patients were recorded as having tumours of 'peripheral' type. The purpose of bronchography is to demonstrate dilated or obstructed bronchi, and bronchography was not often regarded as contributory in establishing the diagnosis of bronchial carcinoma. There are too many other causes, of which mucus is the commonest, of obstruction of bronchi beyond the range of bronchoscopic vision to justify accep- $\stackrel{\infty}{\infty}$ tance of bronchographic evidence of peripheral $\vec{O}$ bronchial obstruction as evidence of carcinoma. Pleural effusion in relation to bronchial carcinoma $\vec{\omega}$ was investigated by thoracentesis, examination of $\stackrel{\curvearrowright}{\rightleftharpoons}$ the pleural liquid both bacteriologically and $x$ histologically, the replacement of the effusion with air, thoracoscopy, and pleural biopsy under iv vision. Biopsies were made of palpable cervical $\vec{\omega}$ lymph nodes, and from other easily accessible

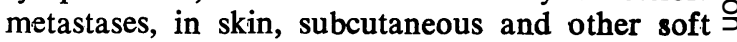
tissues, and in muscle. Sputum was routinely examined for tumour cells, but often not by an expert specifically trained in the techniques in- $\stackrel{\widehat{S}}{\Im}$ volved. Symptoms, such as bone pain and

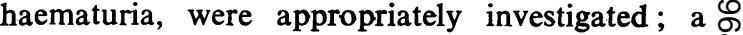
search was not routinely made in symptomless $\infty$ patients for cryptic metastases, by radiographic skeletal surveys or by routine pylography. Special examinations, such as pneumomediastinography, were occasionally undertaken in unusual circumstances but were never a part of routine investigation. Respiratory function, in particular the forced expiratory volume, was measured in patients in whom surgical management was contemplated, but was rarely found more informative than the clinical assessment of exercise tolerance by climbing stairs. An electrocardiogram was made preoperatively in all patients over the age of 40 years. Routine pre-operative chest radiographs made on $\dot{0}$ the day before operation were usually antero- 3 . posterior films made with portable radiographic $\delta$ apparatus with the patient in bed, to provide a 3 more precise base-line against which to judge the 의 post-operative films. A search for tubercle bacilli was made in serial specimens of sputum from all patients.

With regard to the radiographic appearances of bronchial carcinoma, in only three of 4,000 patients were postero-anterior and lateral radio- $\omega$ graphs normal-repeatedly normal and normal to a number of independent experts. Presentation in 0 these three patients was with haemoptysis, in one $\mathbb{D}$ associated with wheeze. The tumours were visible at bronchoscopy, in a main bronchus in all, and 7 also in the trachea in one. Tubercle bacilli were found in the sputum of nearly $2 \%$ of patients with pulmonary carcinoma, and cavitated pulmonary $\stackrel{\unrhd}{\circ}$ túberculosis was recognized radiographically as a concomitant of bronchial carcinoma in $1 \%$ of 8 
patients, bilaterally and a bar to surgical management of carcinoma in some. The radiographic changes of pneumoconiosis were commonplace, and the radiographic features of progressive massive fibrosis were recognized in $1 \%$ of patients. Pleural effusion was a concomitant of bronchial carcinoma in $10 \%$. Obstructive emphysema, the consequence of bronchial carcinoma, was uncommon.

In $7 \%$ of patients with bronchial carcinoma there were related cardiovascular abnormalities, sufficiently gross in one-tenth of these to preclude surgical management. Operative mortality was quadrupled in those with systemic hypertension with electrocardiographic changes, or with a history of angina on effort, or in those who had previously had a myocardial infarct. Of those patients in whom cardiovascular disease and bronchial carcinoma coexisted, who were managed surgically and who died in convalescence, half died from an acute cardiovascular episodemyocardial infarction-or in acute congestive cardiac failure.

Of all patients with bronchial carcinoma, 55\% were unsuitable for surgical management when first they were seen, most because of clinical, radiographic, fluoroscopic, or bronchoscopic evidence of metastases and the remainder because of evidence other than metastases of unsuitability for surgical management or because of death during investigation. The remaining patients $(45 \%)$ were regarded after investigation as suitable for exploratory thoracotomy with a view to resection of pulmonary carcinoma. Nearly one-fifth of these patients, $8 \%$ of the total, were found to have a tumour, the resection of which was technically impossible or therapeutically pointless; and pulmonary resection was completed in approximately $37 \%$ of the total. Pneumonectomy was undertaken twice as often as lobectomy.

The pulmonary tumour in about $55 \%$ of patients with bronchial carcinoma was squamous in type. Undifferentiated tumours, including 'oatcell' carcinoma, accounted for approximately $40 \%$ of the series. Adenocarcinoma was relatively uncommon and in most series accounts for less than $10 \%$ and in this series for no more than $5 \%$ of the total. Alveolar-cell or bronchiolar carcinoma is rare and accounted for less than $1 \%$. In any large series mixed tumours are common-that is, tumours in which a biopsy from the primary may be called squamous, sections of the whole tumour may show different histological varieties, mediastinal or cervical glandular metastases may be undifferentiated, and an hepatic or cerebral metastasis found at necropsy may show the histological morphology of, say, adenocarcinoma. All possible permutations of the histological varieties may be found in the same primary tumour and in metastases. Oat-cell carcinoma is said to be the tumour the histological appearance of which is most consistent throughout the primary and in metastases.

Among those tumours found suitable for management by pulmonary resection, most were squamous; among those tumours found unsuitable for management by exploratory thoracotomy, the number of undifferentiated tumours was in excess of the number of squamous tumours. The operative mortality of exploratory thoracotomy without pulmonary resection was $6 \%$. Nearly three quarters of patients found by exploration to have an irresectable pulmonary carcinoma had died within a year of operation. Nearly half the patients, in whom management of pulmonary carcinoma was by pneumonectomy, were found to have hilar glandular metastases. The operative mortality of pneumonectomy was $12 \%$, and the operative mortality rate increased with age and was $20 \%$ in those 65 years or older. The operative mortality for right pneumonectomy was nearly twice that for left pneumonectomy. The three common causes of death in the post-operative period after pneumonectomy were pulmonary infection, coronary thrombosis, and pulmonary embolism, together accounting for three-quarters of the deaths in this group. The survival rate in the first three years after pneumonectomy for pulmonary carcinoma was significantly higher in those for whom resection was undertaken for squamous carcinoma than in those for whom resection was undertaken for undifferentiated carcinoma. Thereafter survival rates for the two types of tumour ran a roughly parallel course. One in every three patients who died from metastases in the first two years after pneumonectomy died with cerebral metastases. Of all patients in whom bronchial carcinoma was managed by pneumonectomy $20 \%$ were alive more than five years after operation. While most of those who survived a long time were not shown to have mediastinal glandular metastases at operation, the finding of metastases in mediastinal glands is not a bar to long survival. Following pulmonary resection for bronchial carcinoma, survival for more than five years is not an insurance against the development of metastases at a later date, or against the growth of a new primary tumour.

In nearly $70 \%$ of those patients in whom bronchial carcinoma was managed by lobectomy, 
the tumour was squamous in type. The overall operative mortality for lobectomy was approximately half that of pneumonectomy and was $14 \%$ in those 65 years and older, and more than $20 \%$ in those $\mathbf{7 0}$ years and older. Right upper lobectomy carried the highest lobectomy mortality; 'sleeve' resection-usually of the right upper lobe with part of the stem bronchus-was a variety of lobectomy relatively free from special complications. The long survival rate after lobectomy for bronchial carcinoma was twice that for pneumonectomy. The fact that lobectomy for carcinoma is less commonly complicated by death in early convalescence and more commonly followed by long survival than is pneumonectomy does not establish lobectomy for carcinoma as a 'better' operation than pneumonectomy. When a pulmonary carcinoma is manageable by lobectomy the prognosis is better, because of the small size of the tumour, which in turn relates to the biology of the tumour and the rate of growth or early, and therefore often chance, recognition, and to the position of the tumour in relation to the pulmonary hilum.

Bronchial carcinoma in a small number of patients was managed by segmental resection. Segmental resection is not regarded as an acceptable alternative to lobectomy in the management of established bronchial carcinoma, and the circumstances in which segmental resection was undertaken were unusual. Pulmonary carcinoma managed by segmental resection was always peripheral in type and the lesion had often been interpreted, by palpation at thoracotomy, as tuberculous on the grounds of hardness, related scarring, or multiplicity of nodules. Occasionally the segment in which the tumour lay was separated from neighbouring segments by an anatomically complete fissure, usually the apical segment of the lower lobe or the lingular segment of the left upper lobe, and in these circumstances it may be judged as effective to manage the tumour by segmental resection as by lobectomy. In rarer circumstances limitation of respiratory reserve, in patients in whom thoracotomy has been undertaken with a view to making the most limited resection possible to establish the diagnosis of carcinoma, may be an indication for management of a peripheral pulmonary lesion by segmental resection. Among patients in whom bronchial carcinoma was managed by segmental resectionand in all these the tumour was small and peripheral-long survival was as common as after pneumonectomy, and late death was more commonly the consequence of coronary artery insufficiency or respiratory failure than of $\underset{\overrightarrow{\vec{S}}}{\vec{P}}$ metastases.

Those patients who presented without symptoms, $\frac{\bar{\sigma}}{\overline{\frac{D}{3}}}$ and with an abnormality detected on a chest $\frac{\bar{\Phi}}{\bar{\sigma}}$ radiograph made for an unrelated purpose, were $\unrhd$ more often suitable for surgical management of $\nRightarrow$ bronchial carcinoma than were those who pre- $\vec{\circ}$ sented because of symptoms, but not all were suitable for surgical management. In this group $\vec{\omega}$ the number with peripheral tumours was higher and the number with undifferentiated tumours $\vec{x}$ lower than among those who presented with symptoms. Approximately one-quarter of those $i$ who presented without symptoms and with an $\vec{\omega}$ abnormality demonstrated on a routine chest $\sigma$ radiograph made for an unrelated purpose were un- 음 suitable for or declined surgical management. $\overrightarrow{-}$ Operative mortality and the rate of irresectability at exploratory thoracotomy-both about $5 \%$ -

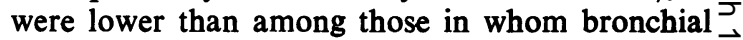
carcinoma had declared itself before radiological $\varnothing$ investigations were undertaken.

Those patients who declined surgical management of a peripheral pulmonary opacity found at routine radiography offered an opportunity, not justifiable in other circumstances, of observing $\stackrel{2}{\not}$ radiographically the varied rate of growth of pul- $\stackrel{\perp}{\Omega}$ monary carcinoma and the time interval between $\vec{\overrightarrow{ }}$ the recognition of a peripheral shadow and the $\frac{0}{3}$ development of symptoms and metastases. Survival $\bar{P}$ time from operation in a small group, who declined the advice offered when first they presented with an abnormal routine film but who later 으 accepted surgical management when they had $\underset{x}{\mathscr{\chi}}$ developed symptoms, was compared with the survival time in those without symptoms who accepted surgical management, and in those who 8 presented for the first time with symptoms and were managed surgically. The number of patients $ᄋ$ in the first group was too small to say anything $>$ more than that the survival time varied over the entire range from long survival with ultimate death without metastases to inoperability with rapid decline despite irradiation, and death with 0 metastases.

Some hazards were attached to routine radio- 0 graphy for whatever purpose. Observer error inco the interpretation of routine miniature films has $\mathbb{\Phi}$ been assessed, and the incidence of error is not $\stackrel{\oplus}{+}$ high, especially if experts collaborate in the inter- $\square$ pretation of routine radiographs. Notwithstanding, $\frac{\vec{P}}{\circ}$ when a patient previously radiographed for routine $\stackrel{\odot}{\Phi}$ purposes presents with new respiratory symptoms $\stackrel{\mathbb{Q}}{\Omega}$ or with a second, now abnormal, film made for 0 similar routine purposes, and the original film, 
passed as normal, is scrutinized with the knowledge gained from recognition of an abnormality in the later film, a similar abnormality in the earlier film is often recognized. This can be of value in reaching a decision on management-for example in making more likely the diagnosis of a chronic inflammatory lesion such as tuberculosis; in assessing the rate of natural growth and, thereby, the prognosis of malignant tumours; and in establishing that other lesions such as chondroadenomata do in fact grow. Furthermore, a patient routinely radiographed at arbitrary intervals, say yearly, who develops respiratory symptoms such as haemoptysis in the interval between radiographs, is inclined to ignore these symptoms in the knowledge that a recent previous film has been passed as normal and another film will be made relatively shortly. But while these are hazards that attach to routine radiography, the overall advantage of this technique has been clearly established. The number of patients found, by chest radiography prompted by the development of a pulmonary complication after an elective surgical procedure other than thoracic, to have a pulmonary lesion other than the acute post-operative one, and probably to which the acute post-operative lesion could be related, is large, and there can never be any justification for not routinely making a chest radiograph before performing any elective surgical procedure in an adult. Where a thoracic surgical unit exists in close relation to general surgical charges and the opportunity for education is taken, a large number of patients will be found in any one year in general surgical wards to have surgical pulmonary abnormalities - an argument in favour of collaboration between the branches of surgery and against the isolation of special units in peripheral hospitals.

Cervical lymph glandular metastasis was the most common single clinical evidence of dissemination of bronchial carcinoma. In approximately $10 \%$ of all patients with pulmonary carcinoma, cervical nodes only on the same side as the primary tumour were the site of metastases. In approximately $2 \%$ cervical glandular metastases were bilateral, and in another $2 \%$ cervical glandular metastases were contralateral. In nearly half those with ipsilateral cervical glandular metastasis this was the only evidence of metastasis on standard clinical and special investigation. Where cervical glands were palpable in patients with bronchial carcinoma, these were found by biopsy to be the site of metastasis in three of every four patients. Scalene node biopsy in patients in whom a gland could not be felt was an unrewarding exercise.
Cervical glandular metastasis from a left pulmonary primary-not only in the lower lobe-to the right side of the neck was not more common than was a metastasis from a right pulmonary primary carcinoma to the left side of the neck. Bilateral cervical glandular metastases were only occasionally the only evidences of spread of bronchial carcinoma, and most patients with bilateral cervical glandular metastases died within a few weeks of investigation.

Axillary lymph glandular metastasis from bronchial carcinoma without chest wall invasion was very rarely the only clinical evidence of metastases and in two patients the primary tumour and the invaded axillary glands were on opposite sides. A radiographic opacity in the vicinity of the azygos vein and inseparable from the mediastinum, in patients in whom the pulmonary primary was right- or left-sided, was good evidence of lymph glandular metastasis and of unsuitability for surgical management. Where the radiographic appearances were those of lymphangitis carcinomatosa, dyspnoea was usually the predominant symptom and death was rapid. Unilateral radiographic features of lymphangitis carcinomatosa were more commonly right- than left-sided ; where the changes were bilateral there was usually a right-sided predominance ; unilateral radiographic features of lymphangitis carcinomatosa were almost always evidence of spread from a primary pulmonary tumour rather than from an extrathoracic primary tumour.

Three per cent of patients found on investigation to have pulmonary carcinoma presented because of cerebral metastases. Hemiplegia, epilepsy, and personality changes were the three most common forms of presentation; speech defects, cerebellar symptoms, and headache were other common presenting symptoms. Only once was it found rewarding to excise a cerebral metastasis and thereafter to manage the pulmonary primary surgically. Where suspicion, however slight, was raised regarding the existence of cerebral metastasis, the presence of which was unconfirmed by investigation, the passage of time, often brief, usually confirmed the suspicion.

Approximately $2 \%$ of patients were found to have osseous metastases as the only evidence of dissemination of bronchial carcinoma at the time of their first investigation ; three in every four of these presented with the complaint of bone pain and without new respiratory symptoms. The commonest sites of osseous metastases were the ribs and the vertebrae. In approximately the same percentage of patients, hepatic metastases were found 
as the only evidence of spread of tumour at the time of first investigation.

Nearly $5 \%$ of patients presented with the clinical features of obstruction of the superior vena cava. More of these were women, of a younger than average age, and the incidence of undifferentiated tumours was higher, than among those who presented in other ways. Obstruction of the superior vena cava and cervical lymph glandular metastases, bilateral disproportionately often, were commonly associated. A right vocal cord palsy in patients with bronchial carcinoma was rare, and when it was found it was usually in association with obstruction of the superior vena cava, a right upper pulmonary carcinoma, and right cervical lymph glandular metastases. It was usually possible quickly to relieve the symptoms and signs of obstruction of the superior vena cava by irradiation; the recurrence of caval obstruction was common and usually a herald of death. Obstruction of the superior vena cava was nearly always associated with a right-sided pulmonary carcinoma, usually in the right upper lobe.

Fluoroscopy demonstrated, in approximately $5 \%$ of patients, that bronchial carcinoma was unsuitable for surgical management-more often because of displacement of the barium-filled oesophagus than because of interruption of a phrenic nerve. Interruption of the left recurrent laryngeal nerve as evidence of mediastinal invasion was found in approximately $4 \%$ of patients. The pulmonary opacity in patients with a left recurrent laryngeal nerve palsy was leftsided in nearly all, and in the left upper lobe or at the left pulmonary hilum in most. Histological confirmation of the diagnosis of bronchial carcinoma was achieved, at the time of their first investigation, in only about one-third of patients who presented with a left cord palsy. Where the histology of the tumour was established, squamous tumours predominated. Survival time from the onset of hoarseness of voice was often long. Irradiation of a pulmonary carcinoma in patients with a hoarse voice did not restore the voice.

Proximal extension of tumour, seen at bronchoscopy and usually established by biopsy, precluded surgical management of pulmonary carcinoma in nearly $5 \%$ of patients. Bronchial carcinoma was judged unsuitable for surgical management when the main carina was seen to be invaded or where the main carina, while not ulcerated, was broad and the main bronchi splayed; where the trachea was involved (unless this involvement was limited to the right lateral wall and this was usually found in relation to a tumour of the right upper bronchus); where the trachea was compressed or scabbard-shaped ; where the tumour would be demonstrated histologically to involve bronchi, usually the main bronchi, of both sides; where tumour obstructed a main bronchus, within $0.5 \mathrm{~cm}$. of the main carina on the right and within $1.5 \mathrm{~cm}$. of the main carina on the left, provided that involvement of this degree of proximity was medial and not only lateral ; and where, by the independent assessment of two experts, a main bronchus was found to be abnormally rigid, an assessment rarely accepted as the only basis for not undertaking surgical management in a patient suitable in all other respects for this form of treatment. Adenocarcinoma rarely extended proximally in the ways outlined. A disproportionately large number of tumours which extended proximally were rightsided.

Invasion of the chest wall, not uncommonly unsuspected clinically, was recognized radiographically in some $3 \%$ of patients. In one-third of these resection was undertaken because chest wall invasion was lateral; in another third the tumour was apical and associated with the features of Pancoast's syndrome; in the last third invasion was medial, either anteriorly or posteriorly.

Where pleural effusion was associated with pulmonary carcinoma, this was routinely investigated by thoracoscopy. This demonstrated the presence of pleural metastases as the only evidence of extra-pulmonary extension of bronchial carcinoma in approximately $2 \%$ of patients. Half the patients with pleural metastases had peripheral tumours, and a disproportionately large number were women. Adenocarcinoma metastasized to the pleura disproportionately often. Most patients with pleural metastases died within six months of the diagnosis having been made, in contradistinction to patients with primary pleural tumours, in whom long survival was not uncommon. Pleural effusion, even if sanguineous, in association with bronchial carcinoma was not synonymous with pleural metastases and was an indication for investigation, not a reason for despair.

Hypertrophic pulmonary osteoarthropathy was a concomitant of bronchial carcinoma in approximately $1 \%$ of patients. In this group of patients the tumour was found disproportionately often to be an adenocarcinoma. The incidence of peripheral tumours and the rate of resectability among 
patients who presented with hypertrophic pulmonary osteoarthropathy was disproportionately high, and the incidence of long survival disproportionately low. Resection of the pulmonary lesion, or irradiation, relieved joint pain. The appearance of intra-thoracic recurrence or metastases in the late post-operative period was, in a small proportion of the patients who presented originally with hypertrophic pulmonary osteoarthropathy, associated with a return of the symptoms and signs of this concomitant of bronchial carcinoma, but this phenomenon was never observed in patients who had not initially presented with hypertrophic osteoarthropathy and who later developed intra-thoracic metastases.

Diminished respiratory reserve constituted the only bar to the surgical management of bronchial carcinoma in approximately $4 \%$ of patients. More than three-quarters of the patients in this group were older than 59 years when first they were seen, whereas in the series as a whole fewer than half the patients were older than 59 years. In elderly patients with poor respiratory reserve and small peripheral tumours, death, more often than not, was with rather than from bronchial carcinoma. In approximately another $4 \%$ of patients there was found a non-metastatic contraindication to surgical management other than diminution in respiratory reserve-for example, extensive pulmonary tuberculosis, chronic asthma, gross pneumoconiosis, bullous emphysema, myocardial disease, advanced age alone, or frailty.

Approximately $2 \%$ of patients who were being investigated for a lesion later established to be a bronchial carcinoma died before investigations could be completed. In many of these the terminal event was not directly attributable to bronchial carcinoma ; at necropsy most had evidence of widespread metastases. Techniques used in the investigation of 4,000 patients with bronchial carcinoma resulted in the death of two patients, a mortality from investigation of $0.05 \%$. 